\title{
On lightening up: improvement trajectories in recovery from depression
}

\section{Gordon Parker}

This article is an extended consideration of a recent editorial (Parker, 1996), and seeks to argue the importance of studying both onset and trajectories of improvement in those with depressive disorders.

\section{Recovery from 'normal' depression}

Some years ago (Parker, 1977) we provided a nonclinical sample with a definition of depression (i.e. 'a significant lowering of mood, with or without feelings of guilt, hopelessness and helplessness, or a drop in one's self-esteem or self-reproach'). All, bar $5 \%$, admitted to such mood states, with a mean frequency of six episodes over the preceding 12 months. For most, such depressive states generally lasted minutes, hours or days, while only $6 \%$ nominated episodes lasting several weeks and $8 \%$ had episodes lasting months. For those who had ever consulted a general practitioner or psychiatrist for depression, such consultation was essentially restricted to those who described their moods lasting weeks or months.

Such findings, replicated and extended in several other non-clinical groups, build to a number of realities. Firstly, that a depressed mood is both a ubiquitous and frequent human experience. Secondly, that while a depressed mood can be quite severe, recourse to help-seeking is often driven more by duration than by severity, a phenomenon addressed by diagnostic manuals such as ICD-10 and DSM-IV imposing a minimum duration. It would appear then that most people accept a depressed mood as a relatively common response to certain vicissitudes of life and that, while it may be severe, it is expected to be transient, and to remit spontaneously and rapidly.

\section{'Clinical' depression}

While a percentage of those who seek professional assistance also have brief depressive disorders, most depressed patients differ as much by their failure to have had a 'natural' or spontaneous remission as by the severity of the mood disorder. This poses a key question - why do some depressed mood states become 'stuck'? As depression is not a homogeneous entity, there is unlikely to be a single answer. For those with a more 'biological' type of depression, there may be an insufficiency of a particular neurotransmitter, so prolonging the depressed mood. By contrast, for those with a more environmentally-induced depression, the stressor (e.g. a grossly dysfunctional marriage) may be persistently 'depressogenic', so constantly fuelling the depressed mood. A third possibility would invoke any number of cognitive explanations, and Teasdale \& Barnard (1993) have explored cognitive models that may "transform an initial mild depressive reaction...into a more severe and persistent state".

In 'normal' depression, subjects will frequently report that their repetitive, reverberating negative thinking and worrying suddenly ceases after several days, often independently of any environmental factor such as the stressor lifting. By contrast, depressed subjects seen in clinical practice appear more commonly to have lost or to lack the capacity for having such reverberating negative

Professor Parker is Professor of Psychiatry, University of New South Wales; Research Director of the Mood Disorders Unit, Prince Henry Hospital; and Director of Psychiatry, Prince of Wales/Prince Henry Hospitals, Randwick 2036, Sydney, Australia. He has published extensively on the depressive disorders. 
cognitions cease. One anxious man with a nonmelancholic depression described how, when depressed, he felt that his brain operated like a gramophone machine. His negative thoughts would go around and around like a long-playing record and, if they were interrupted by some moodlifting event, relief would only be temporary and the 'needle' would drop back onto the record where it had left off. At such times, it generated 'the welling feeling which wears me down'. For him, the introduction of an effective antidepressant drug was influential, as improvement was no longer associated with an incessant need for introspection, in that it was 'no longer the natural thing to do' and with that change came a sense of detachment. Another patient described improvement in similar terms, stating that the drug made him feel, when it became effective, 'care less ... not careless but care less', sidelining his preoccupation with reverberating negative thoughts.

\section{A difference between 'normal' and 'clinical' depression}

If we accept the concept of 'normal' depression as being transient and self-limiting, then it indicates that there would be benefit in understanding factors that trigger spontaneous or self-limiting improvement, and which appear to be largely absent in those who present with clinical depressive conditions, leaving the individual cognitively 'stuck'. This article will consider several recent studies that examine trajectories in recovery patterns from depression, and pursue several clinical implications.

\section{Trajectories in a standard controlled antidepressant drug trial}

Figure 1 illustrates the relatively standard manner in which placebo-controlled antidepressant drug trial data are reported, with changes in Hamilton depression scores (Hamilton, 1960) being examined over 4-6 weeks. Such plots allow three broad conclusions. Firstly, there is a significant placebo response for those receiving an antidepressant. Secondly (at least in drug trials), the efficacy of antidepressant drugs is not striking when the efficacy is represented by the width of the band between the trajectories for the active drug and

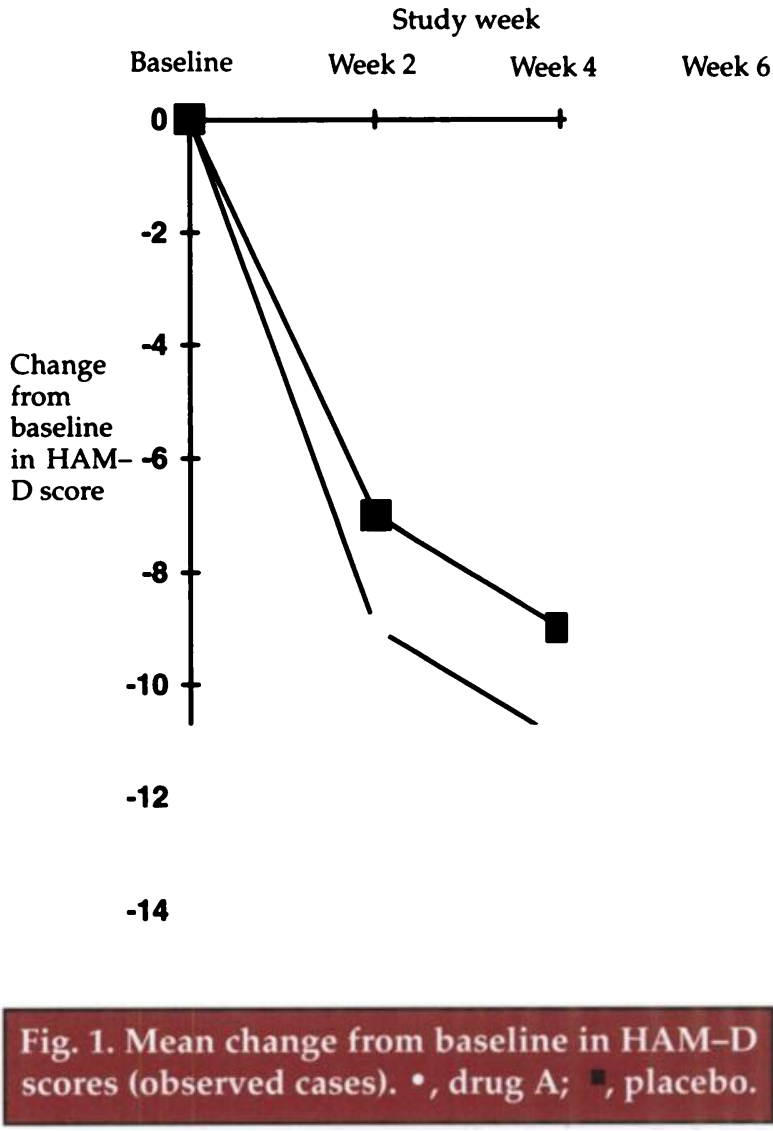

placebo. While this issue is not central to our present enquiry, it is worthy of some note. Narrowness of the band makes definitive assessment of the effectiveness of any new antidepressant difficult for the pharmaceutical companies as they develop their drugs. As a consequence, some effective antidepressant therapies may be aborted before their effectiveness has been demonstrated. Conversely, antidepressant drugs which appear effective during the development stage, may turn out to be less effective in clinical practice when the 'true antidepressant effect' is able to be quantified more accurately.

A paper by Counsell et al (1994) describes the general concern. They note a common scenario: a single small trial with very favourable results for a new therapy is highly likely to act as a 'hypothesis generator' in a series of other randomised control studies. The latter are often small, exploratory and highly susceptible to chance. When treatment effects are slight (in comparison to placebo), it is possible to end up with a number of trials or studies showing a significantly greater than average treatment effect due purely to chance. Counsell and colleagues concluded that we need to be extremely cautious in interpreting the results of individual clinical trials, because of the potentially extreme 
effects of chance on individual studies, and that the conclusions of any systematic review should not be based on a selection of studies but on all the randomised evidence. We need to respect this general proposition when considering the effectiveness of antidepressant drugs, with additional reasons put shortly.

Returning to Fig. 1, a third point requires emphasis. When the effectiveness of the antidepressant drug compared to the placebo is compared at differing time points (a standard procedure in drug trials), it is rare for a statistically significant difference to be evident before at least week four, and often before week six. As noted by Montgomery (1995) "conventional trial designs demonstrating the efficacy of an antidepressant drug have not determined the significant difference in mean response between active drug and placebo reliably before a $4-6$ week period and this is the length of study recommended for the demonstration of acute efficacy in the guidelines of the European Union". This third point alone appears to have been extremely influential in encouraging the interpretation that antidepressant drugs take several weeks to become effective, an issue closely considered in the remainder of this paper.

\section{A Zurich study}

In 1993, a Zurich-based group published an interesting and provocative paper (Stassen et al, 1993) which both challenges long-standing assumptions about delayed onset of antidepressant action and also raises questions as to what might trigger onset of improvement in those receiving antidepressant medication. Their principal data set was derived in a multicentre study, involving a double blind trial of more than 400 moderately depressed patients receiving either oxaprotiline (a selective noradrenaline uptake inhibitor), amitriptyline or a placebo. Subjects were subsequently described as 'responders' 'or non-responders', with a responder being defined as having had a $50 \%$ or greater reduction in the Hamilton depression score at four weeks. Additionally, subjects were assigned as 'improvers' or 'non-improvers', with an 'improver' being an individual who had shown a $20 \%$ reduction in Hamilton depression scores at whatever stage of the trial.

When group data (for the three interventions) were plotted (as in Fig. 1) and analysed, amitriptyline, but not oxaprotiline, was superior to placebo. However, when a survival analysis was undertaken (examining, in this instance, the time to onset of improvement (and which took into consideration those who withdrew due to lack of therapeutic response), a quite different picture emerged. Now, oxaprotiline was more effective than placebo, although not as distinctly as for amitriptyline ${ }^{1}$. The different impression, in comparison to the group data analyses, emerged due to differential drop-out rates across the three interventions (i.e. $46 \%$ for placebo, $19 \%$ for oxaprotiline, and $9 \%$ for amitriptyline) and by withdrawals occurring earlier in the placebo group than for those taking the two active drugs.

Other analyses were of key relevance to an issue pursued in this paper. When the group data for the three interventions were 'deconstructed' into separate responder and non-responder groups, the plotted trajectories showed an interesting pattern. For responders, the time courses and trajectories of improvement were exactly the same, whether receiving oxaprotiline, amitriptyline or placebo. The only difference between the active drug and placebo groups was that, for those receiving an active drug, they were less likely to be a trial dropout and more likely to be in the responder rather than the non-responder group. The authors speculated whether depressed patients may have a 'biological predisposition' to be a responder or not, and that antidepressant drugs appear to act by converting a percentage of 'non-responders' to 'responders', so triggering and perhaps maintaining the conditions required for improvement. Additionally, they concluded that, once triggered, the time course of improvement was identical for those receiving either an antidepressant or the placebo, a conclusion raising questions about the nature of 'triggering' factors.

In a subsequent conference presentation (Stassen \& Angst, 1994), further data were produced challenging the mythology that there is a significant response lag associated with antidepressant medication. The authors suggested that, for those who were trial responders, improvement emerged within the first five days, again whether the improvers were taking an antidepressant drug or placebo. At that conference presentation, the authors stated that when similar analytic strategies had been applied to other antidepressant trial data bases, early onset (within the responders) has again been demonstrated across a range of differing antidepressant drug types. A similar conclusion reported earlier by Small et al (1981) will be noted shortly.

1. Efficacy, improvement and response are terms with differing connotations. Their loose application can distort interpretation of antidepression therapies. 


\section{Other challenges to the concept of delayed onset}

As noted by Gershon (1995), "It has been an established belief that pharmacotherapy with antidepressant agents takes several weeks, 2, 4, or 6 to produce their therapeutic activity". Once accepted, the belief has invited explanations. The supposed delay has been attributed to a range of neurobiological and pharmacokinetic variables, including the need to achieve a steady state therapeutic dose. Charney et al (1981) suggest that the delayed response reflects the time required for induction of one or more receptor modifications (e.g. down regulation). Yet, this generally assumed slow or delayed onset of action of antidepressants is, in fact, contrary to the original report on imipramine (by Kuhn, 1957). As the belief is probably most entrenched in relation to antidepressant drug therapy, we should pursue reasons there, although challenges have existed in the literature for a while.

In 1981, Small et al reported analyses generating conclusions akin to the recent Zurich reports. When grouped data were examined in their study, the formal response rates were $70 \%$ for ECT, $57 \%$ for imipramine, $47 \%$ for trazodone, and $24 \%$ for placebo. Thus, when examined in the orthodox manner (i.e. with group data being plotted), all three active treatments appeared to be superior to placebo, albeit with suggested differential efficacies. When, however, the authors re-plotted the data set for those who were trial 'responders' (i.e. again defined by a $50 \%$ or greater decrease in Hamilton scores by the end of the study), 'all statistical separation between individual treatments and placebo was lost' (Derivan, 1995). As with findings from the Zurich study, identical improvement patterns were evident for responders in each group, and, as observed by Derivan (1995), 'the data suggested most of the treatment effect occurred during the first week of therapy'.

Katz et al (1987) published data nearly a decade ago indicating that responders to tricyclics showed improvement in the first week, most distinctly in disturbed affect, and cognitive function (i.e. thinking, concentration). Early improvement in sleep, however, had no relevance to overall responder status. Recently, Tollefson \& Holman (1994) have reported a meta-analysis comparing improvement patterns in 962 patients receiving fluoxetine and 485 receiving placebo. The large sample size allowed a statistically significant difference favouring fluoxetine to be demonstrated in week one for cognitive disturbance and psychomotor retardation; while anxiety and somatisation separated in the next week; with improvement in sleep disturbance favouring fluoxetine by week two but taking up to week six to show statistical separation.

\section{Does ECT have a response lag?}

A response lag is also commonly described for ECT, although Scott \& Whalley (1993) have argued that few ECT studies have been adequately designed so as to allow clarification of when onset of improvement occurs. They held that preliminary empirical studies neither supported delayed onset of the effect, nor the view that little improvement occurs early during a treatment course. In a subsequent empirical study (Rodger et al, 1994), early response was both demonstrated and quantified, in that improvement after the first three treatments of bilateral ECT was six times greater than that occurring over the remainder of the course.

\section{Early onset in the absence of any antidepressant therapy}

We have similarly described the establishment of an early improvement trajectory in depressed subjects not treated with any antidepressant medication. In separate samples of depressed subjects (all diagnosed as having a non-melancholic type of depression), we required subjects to complete Lubin (1965) adjective check-lists every 6 days (using the 'equivalent' form strategy to overcome practice effects associated with repetition of a single inventory), and examined improvement on other depression inventory scores at 6 and 20 weeks.

In a sample of 43 depressed patients assessed shortly after referral to a psychiatrist (Parker et al, 1985), improvement in Zung Depression Scale scores (the key measure of improvement) (Zung, 1964) at both 6 and at 20 weeks was predicted by the drop in Lubin scores on each occasion of testing, and was evident as early as the first assessment on the sixth day (Pearson's $r=0.52$ and 0.44 ) and most clearly by the 18 th day ( $r=0.60$ and 0.62 ). In a sample of untreated but significantly depressed community subjects who volunteered for a research interview (Parker \& Blignault, 1985), improvement in Zung depression scores at 6 and at 20 weeks was predicted by the degree of improvement in Lubin scores, again as early as the 
Box 1. The 'myth' of delayed onset may reflect:

Failure to recognise that improvement trajectories are different for responders and non-responders

Ratings and clinical assessments too infrequent in the first fortnight

Failure to concede separate clinical subtypes

Failure to distinguish between response and improvement

Artefactual influences

Treatment dose inefficiency

sixth day $(r=0.19)$ but most clearly by the second and third assessments at 12 and 18 days respectively. Predictably, improvement was more pronounced in the treated clinical sample than in the untreated community volunteers (being $24 \%$ and $9 \%$ respectively at 6 weeks).

Nevertheless, for those who did improve in each sample, the trajectory for improvement appeared to be set early, being evident at the initial six-day review. Such data suggest that, in those whose depressive disorders respond within a short period (i.e. 4-6 weeks), whether receiving an antidepressant drug, a non-pharmacological intervention from a psychiatrist or even little more than a research interview, improvement is usually evident in the first week.

\section{Why have we accepted the myth of delayed onset?}

A number of reasons can be noted. First, as described earlier, the standard trial strategy of plotting group data for all those receiving a particular intervention (be it an active drug, ECT or placebo) disguises the reality that, within any group, there will be subsets of 'responders' and 'non-responders'. This finding together with differential drop-out effects for those receiving active treatment and placebo, together with some accepted drug study procedures such as incorporating, for trial drop-out subjects, 'last observation carried forward' data, can all confound interpretation. Again, as demonstrated in Fig. 1, a narrow band of efficacy between an active antidepressant intervention and a placebo may only achieve statistical significance after several weeks, but this does not mean that improvement has not commenced. The inclusion of non-responders contributes considerably to the time required to demonstrate differentiation, for as noted by Laska \& Siegel (1995), "Not everyone who received the treatment has onset, and for those who do not, the time to onset has no meaning".

A second, more minor, issue. Most trial observations are based on weekly assessments of aggregated responders and non-responders, rather than more frequent ratings, so reducing our capacity to detect actual onset of improvement when it occurs.

Third, most trials do little to respect the heterogeneity of depression, so that the common inclusion criterion of 'major depression' may result in a mix of quite differing depressive disorders being represented in the sample. For example, there may be an admixture of those with (i) brief reactive disorders, (ii) major depression superimposed on a more chronic dysthymic-type of disorder and (iii) melancholic depression. Each of those disorders is likely to have quite differing intrinsic and treatmentspecific trajectories of improvement. For instance, it has been estimated by Fairchild et al (1986) that patients with melancholic and non-melancholic depression have quite differing placebo response rates (i.e. $6 \%$ and $54 \%$ respectively) and it is generally recognised (e.g. Rush \& Weissenburger, 1994) that melancholia is more responsive than non-melancholic depression to antidepressant medication. As Montgomery (1995) has observed, a clear differential drug-placebo difference would be more likely if entry criteria limited consideration to in-patients with melancholia. The converse could also be noted - if any trial includes those with brief reactive disorders (and/or others with a high chance of spontaneous remission), then there would be greater difficulty in demonstrating any differential drug-placebo difference. As a consequence, any drug trial that groups intrinsically rapid and slow remitters, as well as aggregating the partial remitter and the non-remitter (for whatever reasons), will give limited information, as the group trajectory clearly subsumes a set of potentially distinctly differing trajectory sub-sets.

Fourth, as stated by Montgomery (1995), 'the myth of the delay in onset of antidepressant action stems largely from the confusion between two related but separate concepts: response and appearance of some early clinical effect'. The strategy adopted by the Zurich workers of operationally defining and distinguishing 'response' and 'improvement' is important and there would be considerable benefit if there could be an agreed lexicon and accepted operational criteria for these two components.

Fifth, there are a number of likely artefactual influences. For instance, when trazodone and bupropion were compared (Weisler et al, 1994) 
using the Hamilton depression measure, trazodone was superior by day seven, but that advantage was no longer evident when the Hamilton insomnia items were removed. Thus, the suggestion that one antidepressant was 'more successful' was due entirely to it being more sedative, and not necessarily being any more successful as an antidepressant.

Sixth, as noted earlier, invalid interpretation may stem from considering only single trials, and conclusions should be based only on large data sets involving all relevant studies. Seventh, an antidepressant may only be effective when prescribed at a certain dose. The wise practice of commencing tricyclic antidepressant drugs at a low dose, before slowly increasing the dose, may clearly delay onset, a phenomenon less likely now that we have antidepressant drugs (e.g. the SSRIs) with a commencing dose that is also likely to be the therapeutic dose (Box 1).

\section{Does early onset of improvement indicate an effective treatment?}

There are a number of difficulties in addressing this issue. Most of the studies considered up to this stage suggest that those who have responded by a defined period (usually 4-6 weeks) will have shown an early onset of improvement. Numerous clinical exceptions could, however, be readily generated, but one example will suffice - the occasional patient who fails to show any improvement or response to a course of ECT until 15 or more treatments. Conversely, early improvement does not necessarily indicate an effective treatment, as it ignores spontaneous remission, placebo response and other explanations. In relation to the last (for the first two possibilities are self-evident), benzodiazepines can induce a rapid response in those with depression, albeit generally not sustained beyond two weeks (Montgomery, 1995).

Some researchers, such as Quitkin et al (1984) have argued that a 'true drug response' is associated with a persistent pattern of improvement, in that once improvement starts, it continues unabated. Quitkin et al defined 'early onset' as improvement commencing in the first two weeks; 'non-persistent response' as improvement with subsequent relapse, and 'delayed onset' as improvement commencing at the end of weeks three to five and continuing without relapse. Turning to the empirical data, Dunlop et al (1990) used rather similar definitions in comparing trajectories of improvement in mildly depressed patients who either received fluoxetine $60 \mathrm{mg}$ per day or placebo. Considering the fluoxetine and placebo responses respectively, an early and persistent response was obtained in $36 \%$ verus 29\%; a delayed persistent response in $21 \%$ versus $6 \%$; and a non-response in $11 \%$ versus $29 \%$, with only the latter two comparisons being significant.

Such data appeared to support the Quitkin view and the common mythology that a true antidepressant drug response is delayed and persistent, and that early response - whether in receipt of active drug or placebo - indicates a placebo response. The Dunlop data, however, were restricted to those with minor depression. The Quitkin view is also challenged by data from the fluoxetine/placebo meta-analysis noted earlier (Tollefson \& Holman, 1994) where specific differential drug effects were noted in the first two weeks. Those authors stated that the available fluoxetine data showed evidence of two persistent patterns - both early abrupt and gradual.

Thus, antidepressant interventions may demonstrate onset rapidly or after a delay. The extent to which early onset is driven by a 'true' antidepressant mechanism or reflects placebo effects and/or spontaneous remission requires clarification, as does the extent to which delayed onset reflects true biological processes and pharmacokinetic issues, or other factors such as the diagnostic sub-type of depression.

\section{Clinical implications}

There is an increasing challenge to the longstanding and wide-spread view that antidepressant drugs and ECT have a significantly delayed onset. Such a generalisation should not be merely replaced by another generalisation - that, if effective, they must always demonstrate improvement within the first week. Certainly, findings from the studies reviewed do suggest that those who 'respond' to such interventions, do tend to show improvement within the first week but, as suggested earlier, clinical observation does indicate a delayed onset for a percentage of responders. We need therefore to clarify mechanisms and clinical guidelines. At this stage, there may be wisdom in suggesting, if a patient does not show some 'improvement' in the first week of antidepressant medication, that the dose may be insufficient or that the particular drug may be ineffective, rather than waiting 3-6 weeks (in case of a delayed onset effect). How the clinician may determine any such 
improvement is, however, problematic, as it may be manifested by an improvement in mood or sleep, or by a reduction in anxiety or across a number of other parameters. Again, as noted earlier, any treatment latency may be very much influenced by the type of depression. Until refined studies have been undertaken which establish the latency of onset and patterns of improvement for separate depressive sub-groups, firm recommendations cannot be drafted.

We clearly need treatment (i.e. drug and ECT) studies to be deconstructed to assess the onset and pattern of improvement in separate subgroups of those who have demonstrated early and delayed improvement patterns, and in separate groups of those having full and partial recovery. The pharmaceutical companies already have large data bases that would allow some of these issues to be pursued, and assist design of refined studies. The implications of such research are important at the clinical and economic level. Perhaps most importantly, we should focus on the issue addressed at the beginning of this article - and examine what causes many depressed patients, as against those with 'normal depression', to get 'stuck' and not experience a spontaneous or rapid remission. As a corollary, we should pursue the suggestion that antidepressant therapies may act by 'kick-starting' a remission, and if so, in which depressive subtypes.

\section{Acknowledgements}

The assistance of Kerrie Eyers and Yvonne Foy in the preparation of this article is gratefully noted.

\section{References}

Charney, D. S., Menkes, D. B. \& Heninger, G. R. (1981) Receptor sensitivity and the mechanism of action of antidepressant treatment. Archives of General Psychiatry, 38, 1160-1180.

Counsell, C. E., Clarke, M. J., Slattery, J., et al (1994) The miracle of DICE therapy for acute stroke: fact or fictional product of subgroup analysis? British Medical Journal, 309, 1677-1681.

Derivan, A. T. (1995) Antidepressants: can we determine how quickly they work? Issues from the literature. Psychopharmacology Bulletin, 31, 23-28.

Dunlop, R. S. Dorenseif, B. E., Wernicke, J. F., et al (1990) Pattern analysis shows beneficial effect of fluoxetine treatment in mild depression. Psychopharmacology Bulletin, 26, 173-180.
Fairchild, C. J., Rush, A. J., Vasavada, N., et al (1986) Which depressions respond to placebo? Psychiatry Research, 18, 217226.

Gershon, S. (1995) Antidepressants: can we determine how quickly they work? Psychopharmacology Bulletin, 31, 21-22.

Hamilton, M. (1960) A rating scale for depression. Journal of Neurology, Neurosurgery and Psychiatry, 23, 56-62.

Katz, M. M., Kowlow, S. H., Maas, J. W., et al (1987) The timing, specificity and clinical prediction of tricyclic drug effects in depression. Psychological Medicine, 17, 297-309.

Kuhn, R. (1957) Uber die behandlung depressiver zustande mit einem Iminopdibenzylderivat. Schweizerische Medizinische Wochenschrift, 87, 1135-1140.

Laska, E. M. \& Siegel, C. (1995) Characterizing onset in psychopharmacological clinical trials. Psychopharmacology Bulletin, 31, 29-35.

Lubin, B. (1965) Manual for the Depression Adjective Check List. San Diego: Educational and Industrial Testing Service.

Montgomery, S. A. (1995) Are 2-week trials sufficient to indicate efficacy? Psychopharmacology Bulletin, 31, 41-44.

Parker, G. (1977) Parental Antecedents to Depression. MD Thesis, University of New South Wales.

- (1996) On brightening up: triggers and trajectories to recovery from depression. British Journal of Psychiatry, 168, 263-264.

- \& Blignault, I. (1985) Psychosocial predictors of outcome in subjects with untreated depressive disorder. Journal of Affective Disorders, 8, 73-81.

-, Tennant, C. \& Blignault, I. (1985) Predicting improvements in patients with non-endogenous depression. British Journal of Psychiatry, 146, 132-139.

Quitkin, F. M., Rabkin, J. G., Ross, D., et al (1984) Identification of true drug response to antidepressants: use of pattern analysis. Archives of General Psychiatry, 41, 782-796.

Rodger, C. R., Scott, A. I. \& Whalley, L. J. (1994) Is there a delay in the onset of the antidepressant effect of electroconvulvsive therapy? British Journal of Psychiatry, 164, 106-109.

Rush, A. J. \& Weissenburger, J. E. (1994) Melancholic symptom features and DSM-IV. American Journal of Psychiatry, 151, 489498.

Scott, A. I. F. \& Whalley, L. J. (1993) The onset and rate of the antidepressant effect of electroconvulsive therapy. A neglected topic of research. British Journal of Psychiatry, 162, 725-732.

Small, J. G., Milstein, V., Kellams, J. J., et al (1981) Comparative onset of improvement in depressive symptomatology with drug treatment, electroconvulsive therapy, and placebo. Journal of Clinical Psychopharmacology, 1 (suppl 6), 62-69.

Stassen, H. H., Delini-Stula, A. \& Angst, J. (1993) Time course of improvement under antidepressant treatment: a survivalanalytical approach. European Neuropsychopharmacology, 3, 127-135.

- \&Angst, J. (1994) Methods of Estimating Onset of Improvement. Paper presented at VII Congress of the European College of Neuropsychopharmacology, Jerusalem.

Teasdale, J. D. \& Barnard, P. J. (1995) Affect Cognition and Change: Re-modelling Depressive Thought. Hove: Lawrence Erlbaum.

Tollefson, G. D. \& Holman, S. L. (1994) How long to onset of antidepressant action: a meta-analysis of patients treated with fluoxetine or placebo. International Clinical Psychopharmacology, 9, 245-250.

Weisler, R. H., Johnston, J. A., Lineberry, C. G., et al (1994) Comparison of bupropion and trazodone for the treatment of major depression. Journal of Clinical Psychopharmacology, 14, 170-179.

Zung, W. W. K. (1964) A self-rating depression scale. Archives of General Psychiatry, 12, 63-70. 


\section{Multiple choice questions}

1 Clinical depression differs from 'normal depression' by

a being generally more severe

b generally lasting longer

c being more likely to show a spontaneous remission

$\mathrm{d}$ being a more common human experience

2 Standard placebo controlled antidepressant drug trials examining group data demonstrate a antidepressant drugs to be highly effective

b early significant differential improvement

c antidepressant drugs to have an earlier onset of improvement than ECT

d antidepressant drugs to have an earlier onset than placebos

3 In standard controlled studies, improvement in those who receive an antidepressant drug

$a$ is more likely than for those receiving a placebo

$b$ is associated with a high drop-out rate

c generally takes 10 days to 3 weeks to be evident

d has a different trajectory to those who improve in response to ECT
4 The following interventions appear to have a significant response lag before showing any antidepressant effect
a ECT
b tricyclic antidepressant drugs
c psychotherapy
d placebos

5 Early improvement after being prescribed an antidepressant drug

a indicates that recovery will occur

b favours the non-melancholic type of depression

c argues against a 'true drug response'

d suggests that the treatment has been effective

MCQ answers

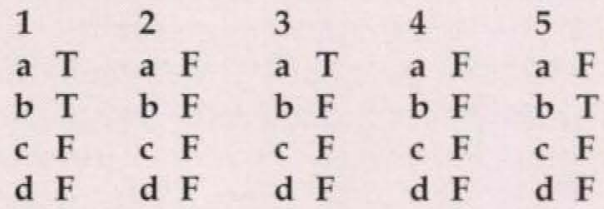

